\title{
Assessing alcohol drinking measures, motives and problems among university students in the UK
}

\section{Mohammed Alghamdi}

Faculty of Arts and Humanities, King Abdul-Aziz University, Kingdom of Saudi Arabia abu400abdullah400@gmail.com

Received: 10 Jan. 2020-02-23

Accepted: 20 Feb. 2020

This study investigated the correlation between university student drinking measures, the motives for drinking and the actual consequences/problems of drinking. It was conducted using quantifiable data collected via online surveys from 126 university student drinkers. The results of the study revealed that the more units, and the greater frequency of consumption, were associated with a higher likelihood of physical and psychological consequences, and a reduction of the quality of life. The results also indicated that the most significant outcome was adverse effects on interpersonal relationships. The greatest motives were conformity, social relationships, and mood enhancements. The results of this study had been discussed.

Keywords: Alcohol consumption, UK university students, effects, motives, measures.

\section{INTRODUCTION}

Excessive alcohol consumption measures are important areas of study, because alcohol is a mind-altering substance ${ }^{(\mathbf{1})}$. Alcohol is also a "mood altering" substance ${ }^{(\mathbf{2})}$. Alcohol consumption can result in dependency, and its harms include health issues and socioeconomic problems for the afflicted, his and her family, and society in general ${ }^{\mathbf{( 1 )}}$ (World Health Organization, 2014. Alcohol consumption can also cause legal problems for the consumer, his/her family and society ${ }^{(3)}$. Alcohol consumption is ranked as the world's fifth disease-causing agent ${ }^{(\mathbf{4})}$. Heavy drinkers tend to share the same behaviours and consequences, internationally ${ }^{(5)}$. Yet an accurate account of alcohol consumption is impossible, since data does not include alcohol made at home, alcohol that was purchased duty-free, and alcohol that was smuggled into a country ${ }^{(4)}$. Therefore, the true extent of alcohol's burden on society is unknown.

\section{Hypotheses}

A review of literature identified several dependent variables that explain youth consumption of alcohol. The dependent variables explaining motives are mood enhancements, socialization, fitting in, entertainment, self-confidence, and so on. The dependent variables explaining the psychological and physical consequences of drinking alcohol, are hangovers, embarrassment, guilt, lost days of work, poor parenting, negative impact on personal, social, and familial relationships, poor sexual performance, obesity or weight gain, and poor physical appearance. The independent variables identified in the literature are age, gender, year of study, religion and nationality as well as drinking levels from moderate to harmful or hazardous or binge drinking. To test the variables and the correlation between the variables, the following hypotheses were tested:

H1: University students are irresponsible alcohol consumers.

$\mathrm{H} 2$ : University students engage in harmful/hazardous/binge alcohol consumption frequently or regularly. 
Mohammed Alghamdi

H3: University students suffer physically harmful effects of frequent, or regular, harmful/hazardous/binge alcohol consumption.

H4: University students suffer psychologically harmful effects of frequent, or regular, harmful/hazardous/binge alcohol consumption.

H5: University students suffer a lower quality of life due to frequent, or regular, harmful/hazardous/binge alcohol consumption.

H6: University students are motivated to consume alcohol for inter- and intrapersonal, conformity, social and mood enhancement reasons.

\section{Excessive Drinking among College/University}

Many countries around the world report hazardous drinking among university students as a major public health concern. One of the public health problems identified is the link between alcohol abuse, and cigarette smoking, together with illicit drug abuse ${ }^{(6)}$. Alcohol consumption in Muslim countries in the Indian "subcontinent", and the "eastern Mediterranean region were especially "low" (7). This suggests that religion is an important factor in the individual's formation of drinking habits. Muslims have established a culture, and habit, that is averse to alcohol consumption ${ }^{(7)}$.

\section{Purpose of the Study}

A review of literature as reported thus far indicates that alcohol consumption among students is high and harmful in nature. As the harmful nature of alcohol consumption among young people remains a problem, there is an urgent need to identify plausible and workable solutions. To get there, a better understanding of the motives, measures and problematic effects of alcohol consumption by the youth population is required. This research will therefore contribute to current knowledge on alcohol consumption by measuring consumption, motivation and problematic effects of consumption among college students. The researcher developed a survey questionnaire for this purpose.

\section{Quantitative.}

\section{METHODS}

The exploratory and descriptive research study was quantitative in nature. A quantitative research design involves the collection of data that is measurable or quantifiable $^{(\mathbf{8})}$. Quantitative research can be either experimental or non-experimental in design, if it produces numeric or measurable data. The non-experimental design usually involves a close-ended survey, with data that can be numerically or statistically calculated. The quantitative research design, will usually contain research questions and/or hypotheses that will be tested through an instrument, such as surveys that collect data accordingly ${ }^{(8)}$.

\section{Instrument.}

In the quantitative design of this research study, a non-experimental, close-ended and multiple-choice survey was used. A close-ended and multiple-choice survey involves questions followed by short or "possible responses" ${ }^{(9)}$. The survey was divided into four parts. The first part of the survey collected demographic data. The second part of the survey collected data on the drinking history of the participants over the two weeks just prior to filling out the survey (TADD). The Third part of the survey collected data on the effects of consuming alcohol on the participants over the last three months (DrInC-2R). The fourth part of the survey collected data on the drinking motives of the participants (DMQ-R). The final part of the survey was the World Health Organization Quality of Life questionnaire (WHOQOL-BREF). 


\section{Assessing alcohol drinking measures, motives and problems among university students in the UK}

\section{Procedure}

The data collection procedure began with establishing the inclusion criteria and selecting a target audience. This was followed by contact and consent. The consent was placed in the survey together with explanations about the research to ensure informed consent. The survey was placed on the Internet. Once the online survey was completed by the requisite number (125) university students with a history of consuming alcohol beverages, the survey was closed to additional participants.

The completed surveys were checked for internal consistency and completeness. The variables and the corresponding responses were entered into the SPSS software, and analyzed using linear regression analysis. In the process, ANOVA was conducted. ANOVA is a process that measures variances between variables. Specifically, ANOVA refers to estimating and evaluating variances ${ }^{(\mathbf{1 0})}$. In simple terms, ANOVA measures the differences within and between groups and determines whether those differences are significant ${ }^{(\mathbf{1 1})}$. For example, this study measures the drinking habits, motivations and consequences for different groups such as male and females and diverse nationalities and religions. In the ANOVA, mean squares, sum of squares and deviations reveal variances between groups and identify whether those variances have any statistical significance. In other words, the ANOVA will determine whether, nationality, age, gender, marital status, and/or religion account for the drinking patterns, motivations and effects.

\section{RESULTS}

Although a total of 125 survey participants were targeted, 126 students responded to the survey. It was obvious from Table (1) that the mean age of the participants was 21.75 years with a standard deviation of 2.39485. Only a minimal number of participants were over the age of 25. Most of the participants were female (90 or $71.4 \%$ ) with only 34 males $(27.0 \%)$. Fifty-five of the participants were in their second year of study with second largest group consisting of 31 in their $1^{\text {st }}$ year. The third largest group of 25 cohorts was in their $4^{\text {th }}$ year. Only 9 and 2 of the participants were in their $3^{\text {rd }}$ and $5^{\text {th }}$ years of study, respectively.

Most of the participants were atheist (57). Christians made up the second largest group of cohorts at 40. There were only five Buddhists; four Agnostics; and 2 Muslims. With regards to nationality, 101 of the participants were European, representing a significant majority. Twelve of the respondents were Asian; four were African, and 3 were North American. 
Mohammed Alghamdi

Table 1. Demographic Variables

\begin{tabular}{|c|c|c|c|c|c|}
\hline Demographic data & Number & $\%$ & Demographic data & Number & $\%$ \\
\hline $\begin{array}{l}\text { Age (Years) } \\
18-25 \\
20 \\
21 \\
26-33\end{array}$ & $\begin{array}{l}112 \\
36 \\
31 \\
8\end{array}$ & $\begin{array}{l}88.9 \\
28.6 \\
24.6 \\
7.2\end{array}$ & $\begin{array}{l}\text { Nationality } \\
\text { African } \\
\text { North American } \\
\text { European } \\
\text { Asian }\end{array}$ & $\begin{array}{l}4 \\
3 \\
101 \\
12\end{array}$ & $\begin{array}{l}3.2 \\
2.4 \\
80.2 \\
9.5\end{array}$ \\
\hline $\begin{array}{l}\text { Gender } \\
\text { Male } \\
\text { Female }\end{array}$ & $\begin{array}{l}34 \\
90 \\
\end{array}$ & $\begin{array}{l}27.0 \\
71.4 \\
\end{array}$ & $\begin{array}{l}\text { Marital Status } \\
\text { Single } \\
\text { Married }\end{array}$ & $\begin{array}{l}115 \\
2\end{array}$ & $\begin{array}{l}91.3 \\
1.6 \\
\end{array}$ \\
\hline $\begin{array}{l}\text { Year of Study } \\
1^{\text {st }} \text { Year } \\
2^{\text {nd }} \text { Year } \\
3^{\text {rd }} \text { Year } \\
4^{\text {th }} \text { Year } \\
5^{\text {th }} \text { Year }\end{array}$ & $\begin{array}{l}31 \\
55 \\
9 \\
25 \\
2 \\
\end{array}$ & \begin{tabular}{l|}
24.6 \\
43.7 \\
7.1 \\
19.8 \\
1.6 \\
\end{tabular} & $\begin{array}{l}\text { Nationality } \\
\text { African } \\
\text { North American } \\
\text { European } \\
\text { Asian }\end{array}$ & $\begin{array}{l}4 \\
3 \\
101 \\
12\end{array}$ & $\begin{array}{l}3.2 \\
2.4 \\
80.2 \\
9.5\end{array}$ \\
\hline $\begin{array}{l}\text { Religion } \\
\text { Atheist } \\
\text { Christian } \\
\text { Buddhist } \\
\text { Agnostic } \\
\text { Muslim } \\
\end{array}$ & $\begin{array}{l}57 \\
40 \\
5 \\
4 \\
2 \\
\end{array}$ & \begin{tabular}{l|}
45.2 \\
31.7 \\
4.0 \\
3.2 \\
1.6 \\
\end{tabular} & & & \\
\hline
\end{tabular}

Descriptive statistics (Table 1) revealed an identifiable pattern where mean averages of TADD days of drinking were 9.3440 and units drank (TADD Units, Heavy Light Drinkers) had a mean average of 1.4298 and a standard deviation of 49724. Standard deviations for TADD Units were high at 61.29052 indicating that there is a significant amount of variance in terms of units of alcohol consumed among the population studied. The standard deviation on WHOQOL Physical (17.51619), WHOQOL Psychological (18.70113) and WHOQOL Environment (18.25995) were also relatively large indicating significant variance in quality of life. The standard deviation for conformity as a reason for drinking, compared to a mean of 8.2177 , is significant because it shows very little variance within the population studied. Inter- and Intra-Personal reliable variables, showed the closest correlation between drinking and motives.

Table 2. Descriptive Statistics

\begin{tabular}{|l|l|l|l|}
\hline & Mean & Std. Deviation & $\mathrm{N}$ \\
\hline TADD_Days & 9.344 & 6.10804 & 125 \\
TADD_Units_Heavy Light Drinkers & 1.4298 & .49724 & 114 \\
TADD_Units & 72.8338 & 61.29052 & 114 \\
TADD_Binges & 4.7632 & 4.55313 & 114 \\
Motives_Social & 15.8293 & 4.88676 & 123 \\
Motives_Coping & 10.7419 & 4.10803 & 124 \\
Motives_Enhancement & 13.0806 & 4.88834 & 3.92938 \\
Motives_Conformity & 8.2177 & 17.51619 & 124 \\
WHOQOL_Physical Health & 110.4065 & 18.70113 & 124 \\
WHOQOL_Psychologi Cal & 80.0331 & 9.42156 & 123 \\
WHOQOL_Social_Relation & 45.4194 & 18.25995 & 121 \\
WHOQOL_Environment & 120.6612 & 3.38746 & 124 \\
DrInc_Physial_Reliable & 4.9238 & 3.43592 & 121 \\
DrInc_InterPersonal_Reliable & 2.6500 & 3.68513 & 105 \\
DrInc_IntraPersonal_Reliable & 3.0481 & 4.27418 & 100 \\
DrInc_Impulse Control_Reliable & 4.7900 & 3.15827 & 104 \\
DrInc_Social Responsibility_Reliable & 2.8416 & & 100 \\
\hline
\end{tabular}




\section{Assessing alcohol drinking measures, motives and problems among university students in the UK}

(TADD): collected data on the drinking history of the participants over the two weeks just prior to filling out the survey

(DrInC-2R): collected data on the effects of consuming alcohol on the participants over the last three months.

(DMQ-R): collected data on the drinking motives of the participants.

(WHOQOL-BREF): World Health Organization Quality of Life questionnaire.

Statistical analyses revealed more specific results. The linear regression produced a line of best fit, showing that the most significant relationship was between two variables e.g Gap Between Christians and Atheists and other Religions (Fig. 1).

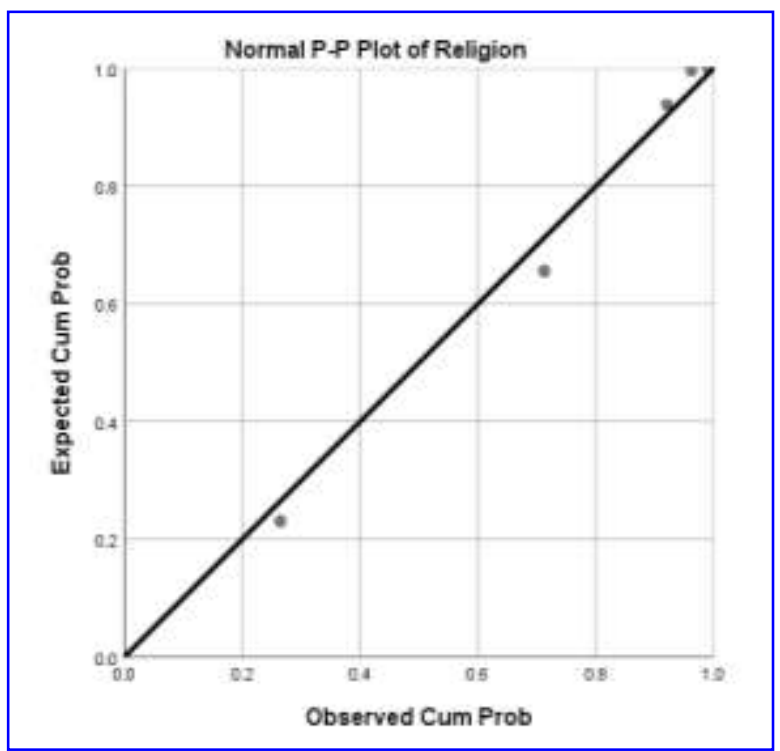

Fig. 1. Religion: Gap Between Christians and Atheists and other Religions

The results revealed that there are a low percentage of respondents reporting satisfaction with their respective physical environment. When we consider the fact that drinking alcohol was found to be linked to mood enhancements, this is important when considering the results of this study indicating that nearly $30 \%$ of the respondents, were binge drinking over the two weeks prior to completing this survey.

\section{DISCUSSION}

The results of the study are consistent with findings in the literature in that there is a correlation between excessive consumption of alcohol, and physical and psychological consequences $^{(\mathbf{1 2})}$. The study results showed a definitive correlation between students who drank excessively, and reports of difficulties with psychical and psychological health outcomes. The age of the respondents and the likelihood that excessive consumption of alcohol was recent development, precluded assumptions about acute health. A study following the respondents' drinking habits over a period of years, and health outcomes would predict whether acute health problems are consistent with excessive consumption over a long period of time.

Based on the results of this study the hypotheses are accepted and rejected as follows: H1: University students are irresponsible alcohol consumers. 
Mohammed Alghamdi

$\mathrm{H} 1$ is rejected as only partially true. Findings in this study support the contention that some university students are responsible drinkers, although far too many students are harmful or hazardous alcohol consumers.

$\mathrm{H} 2$ : University students are harmful/hazardous/binge alcohol consumers.

$\mathrm{H} 2$ is accepted as partially true since a statistically significant number of university students are harmful/hazardous/binge alcohol consumers. The fact that not all university students are harmful/hazardous/binge alcohol consumers is irrelevant. If there is a statistically significant harmful alcohol consumption population, there is an issue for further research and further action.

H3: University students suffer physically harmful effects of alcohol consumption.

$\mathrm{H} 3$ is accepted as confirmed since students participating in the study reported physical health outcomes relative to a decline in quality of life due to the harmful effects of regular binge alcohol consumption.

H4: University students suffer psychologically harmful effects of regular binge alcohol consumption.

$\mathrm{H} 4$ is accepted as confirmed since students participating in the study reported psychological health outcomes relative to a decline in quality of life due to the harmful effects of regular binge alcohol consumption.

H5 University students suffer a lower quality of life due to regular or frequent alcohol consumption.

H5 is also accepted as confirmed since students participating in the study reported a lower quality of life due to frequent or regular harmful/hazardous/binge alcohol consumption.

H6 is accepted as confirmed because, students were motivated to drink alcohol for inter- and intrapersonal, social and conformity reasons.

\section{Conclusion}

Concerns about the public health issues associated with university students' excessive consumption of alcohol have been confirmed by this study. Although the student population participating in the study was primarily females, the results are disturbing all the same. First, the results indicate that female students are increasingly engaging in excessive alcohol consumption. Secondly, the study confirms that excessive alcohol consumption on UK university campuses continues to occur.

The harmful effects of excessive alcohol consumption are undisputed in the literature. Similarly, the harmful effects of excessive alcohol consumption are also confirmed by this study although, acute health effects were not identified. Even so, the young ages of the participants do not exclude the acute health effects of prolonged, excessive drinking of alcohol. It can be assumed that should the excessive alcohol consumers identified in this study continue to imbibe excessively, acute physical and psychological health problems will occur.

Given the fact that students participating in this study reported social and conformity motivating factors, it is reasonable to assume that alcohol consumption is institutionalized on college campuses in the UK. It is obviously necessary for university campuses to address this issue. This study is important because it supports findings in the literature indicating how much university students drink, their motivations and the consequences of excessive drinking. With support for findings in the literature, we can confirm that university consumption is a problem that should be addressed. Based on the results of this study, recommendations are made for university campuses to take measures that eliminate the institutionalization of alcohol consumption. 


\section{Assessing alcohol drinking measures, motives and problems among university students in the UK}

\section{Strengths of the Study}

The strengths of the study are found in the focus on a sample of university students actively on campus with a history of alcohol consumption. Therefore, the researcher could collect direct evidence of alcohol consumption patterns, outcomes and motivations among university students in the UK.

\section{Suggestions for further Research}

To gain more insight into the measures, effects and motivation of university students' consumption of alcohol, a more varied student population should be targeted. Such a student population would include a more balanced gender, marital status, religion and nationality. This balance would provide more insight into the correlation between demographic data and university students' consumption of alcohol. By taking this approach, external influencing factors such as nationality or religion can be separated from the university influences. This would provide university personnel with more accurate and effective policies for controlling excessive alcohol consumption on its campuses. Further research on university students' alcohol consumption should also include a comparative study in which the drinking culture of youth who are not university students are compared. A comparative study of this nature will enable researchers to determine whether the drinking culture of the youth is specific to university conditions, or simply a problem associated with the young culture. This kind of research findings can direct both policy makers and university administrators on the appropriate policies and practices design to reduce or eliminate a youth culture determined to engage in excessive alcohol consumption.

\section{REFERENCES}

1. World Health Organization. (2014). Global Status Report on Alcohol and Health. Retrieved from:

a. http://www.who.int/substance_abuse/publications/global_alcohol_report/msb_gsr_2014_1.pdf

2. Nordegren, T. (2002). The A-Z Encyclopedia of Alcohol and Drug Abuse. Parkland, FL: Brown Walker Press.

3. Maltzman, I. (2008). Alcoholism: Its Treatments and Mistreatments. London: World Scientific Publishing Co. Ltd.

4. Monteiro, M.G.; Rehm, J. and Shield, K.D. (December 2015). Alcohol Consumption: An Overview of International Trends. Elsevier Reference Module in Biomedical Sciences, 1-14.

5. Aboud, F.E. (1998). Health Psychology in Global Perspective. Thousand Oaks, CA: SAGE Publications, Inc.

6. Davore, M.P.; Shiely, F.; Byrne, M. and Perry, I.J. (2015). Hazardous Alcohol Consumption among University Students in Ireland: A Cross-Sectional Study, Open BMJ, $10,1-9$.

7. Ahlstrom, S.K. and Osterberg, E.L. (2004/2005). International Perspectives on Adolescent and Young Adult Drinking. Alcohol Research and Health, 28(4): 258-268.

8. Creswell, J. W. (2014). Research Design: Qualitative, Quantitative and Mixed Methods Approaches (4th ed.) Scientific Research An Academic publisher.

9. Gideon, L. (2012). Handbook of Survey Methodology for the Social Sciences. New York, NY: Springer.

10. Muller, K.E. and Fetterman, B.A. (2002). Regression and ANOVA: An Integrated Approach using SAS Software. Cary, NC: SAS Institute Inc.

11. Miller, R.G. (1998). Beyond ANOVA: Basics of Applied Statistics. CRC Press LLC 
Mohammed Alghamdi

12. Institute of Alcohol Studies. (August 2013). Alcohol Consumption: Factsheet. Retrieved from:

http://www.ias.org.uk/uploads/pdf/Consumption\%20docs/Alcohol\%20consumption\%20f actsheet\%20August\%202013.pdf

$$
\text { تقييم مقايس شرب الكحول والدوافع والمشاكل بين طلاب الجامعة }
$$

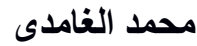

كلية الآداب و العلوم الانسانية-جامعة الملك عبد العزيز ـالمملكة العربية السعودية

تتتاول هذه الدر اسة العلاقة بين مقايس شرب الكحول لألى الطلاب في الجامعة ، و الدو افع ، و النتائج / المشاكل

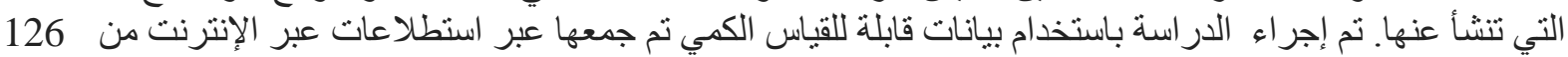

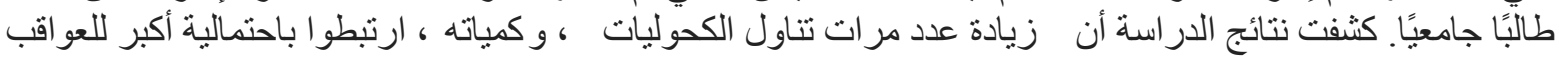

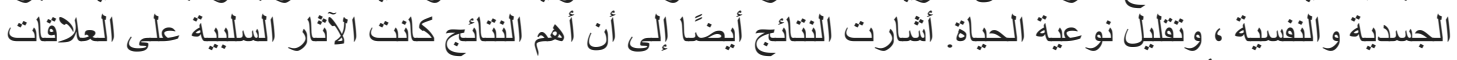
الثخصية. وكانت أعظم الدو افع هي المطابقة والعلاقات الاجتماعية وتحسين المز اج. وقد تم مناقثة نتائج هذه الدر استة الثات. 